\title{
CONTRIBUTIONS TO THE THEORY OF MARKOV CHAINS. II
}

\author{
BY \\ K. L. CHUNG(1)
}

Introduction. This is a sequel to my paper [1]. The present developments are largely independent of the previous results except in so far as given in the Appendix. Theorem 1 shows a kind of solidarity among the states of a recurrent class; it generalizes a classical result due to Kolmogorov and permits a classification of recurrent states and classes. In $\$ 2$ some relations involving the mean recurrence and first passage times are given. In $\$ \$ 3-5$ sequences of random variables associated in a natural way with a Markov chain are studied. Theorem 2 is a generalized ergodic theorem which applies to any recurrent class, positive or null. It turns out that in a null class there is a set of numbers which plays the role of stationary absolute probabilities. In the case of a recurrent random walk with independent, stationary steps these numbers are all equal to one and the result is particularly simple. Theorem 3 shows that the kind of solidarity exhibited in Theorem 1 persists in such a sequence; it leads to the clarification of certain conditions stated by Doblin $\left({ }^{2}\right)$ in connection with his central limit theorem. Using a fundamental idea due to Doblin, the weak and strong laws of large numbers, the central limit theorem, the law of the iterated logarithm, and the limit theorems for the maxima of the associated sequence are proved very simply. Owing to the great simplicity of the method it is the conditions of validity of these limit theorems that should deserve attention. Among other things, we shall show by an example that a certain set of conditions, attributed to Kolmogorov, is in reality not sufficient for the validity of the central limit theorem. Furthermore, conditions of validity for the strong limit theorems and the limit theorems for the maxima are obtained by a rather natural strengthening of corresponding conditions for the weak limit theorems. A word about the connection of these conditions with martingale theory closes the paper.

1. The sequence of random variables $\left\{X_{n}\right\}, n=0,1,2, \cdots$, forms a denumerable Markov chain with stationary transition probabilities. The states will be denoted by the non-negative integers $\left({ }^{3}\right) 0,1,2, \cdots$. The $n$-step transition probability from the state $i$ to the state $j$ will be denoted by $P_{i j}^{(n)}\left(P_{i j}^{(1)}=P_{i j}\right)$. Thus we have

$$
P_{i j}^{(n)}=P\left(X_{m+n}=j \mid X_{m}=i\right)
$$

Presented to the Society, September 1, 1953; received by the editors April 17, 1953.

(1) This research is done on an Air Research and Development Command Project.

(2) Doeblin.

(3) We note that in [1] the states are the positive integers. 
for all integers $m \geqq 0$ for which the conditional probability is defined. In the following we shall simply write $m=0$ in such formulas. The initial probabilities, namely the distribution of $X_{0}$, are fixed but arbitrary.

The notations used below are the same as in [1]. In particular

$$
\begin{aligned}
{ }_{k} P_{i j}^{(n)} & =P\left(X_{n}=j, X_{v} \neq k, 1 \leqq v<n \mid X_{0}=i\right), \\
F_{i j}^{(n)} & =P\left(X_{n}=j, X_{v} \neq j, 1 \leqq v<n \mid X_{0}=i\right), \\
{ }_{k} F_{i j}^{(n)} & =P\left(X_{n}=j, X_{v} \neq j, \neq k, 1 \leqq v<n \mid X_{0}=i\right), \\
m_{i j}^{(p)} & =\sum_{n=1}^{\infty} n^{p} F_{i j}^{(n)},{ }_{k} m_{i j}^{(p)}=\sum_{n=1}^{\infty} n^{p}{ }_{k} F_{i j}^{(n)}, \\
{ }_{k} P_{i j}^{*} & =\sum_{n=1}^{\infty}{ }_{k} P_{i j}^{(n)}, \\
F_{i j}^{*} & =\sum_{n=1}^{\infty} F_{i j}^{(n)},{ }_{k} F_{i j}^{*}=\sum_{n=1}^{\infty}{ }_{k} F_{i j}^{(n)} .
\end{aligned}
$$

We shall confine ourselves to one recurrent class, hence all $F_{i j}^{*}=1$.

THEOREM $1\left(^{4}\right)$. Let $p$ be a fixed positive real number. If

$$
m_{i j}^{(p)}<\infty, \quad m_{j i}^{(p)}<\infty
$$

for a pair of states $i$ and $j$ (distinct or not) in a recurrent class, then the same is true for every pair of states (distinct or not).

Proof. We have, by the usual arguments, if $i \neq j$ :

$$
\begin{aligned}
& F_{i i}^{(n)}=\sum_{v=1}^{n-1}{ }_{i} F_{i j}^{(v)} F_{j i}^{(n-v)}+{ }_{j} F_{i i}^{(n)}, \\
& F_{i j}^{(n)}=\sum_{v=1}^{n-1}{ }_{j} F_{i i}^{(v)} F_{i j}^{(n-v)}+{ }_{i} F_{i j}^{(n)} .
\end{aligned}
$$

Multiplying through by $n^{p}$ and summing from $n=1$ to $n=\infty$, we obtain

$$
\begin{aligned}
& m_{i i}^{(p)}=\sum_{v=1}^{\infty}{ }_{i} F_{i j}^{(v)} \sum_{s=1}^{\infty}(s+v){ }^{p} F_{j i}^{(s)}+{ }_{j} m_{i i}^{(p)}, \\
& m_{i j}^{(p)}=\sum_{v=1}^{\infty}{ }_{j} F_{i i}^{(v)} \sum_{s=1}^{\infty}(s+v)^{p} F_{i j}^{(s)}+{ }_{i} m_{i j}^{(p)} .
\end{aligned}
$$

(i) We shall show that (1) implies that

(4) Added in proof. A partial result, for integral values of $p$, was proved independently by J. L. Hodges and M. Rosenblatt, Recurrence-time moments in random walks, Pacific Journal of Mathematics vol. 3 (1953) pp. 127-136. 


$$
m_{i i}^{(p)}<\infty .
$$

If $i=j$ there is nothing to show. Otherwise from (3) we obtain, since $(s+v)^{p}$ $\leqq 2^{p}\left(s^{p}+v^{p}\right)$,

$$
m_{i i}^{(p)} \leqq 2^{p} \sum_{v=1}^{\infty}{ }_{i} F_{i j}^{(v)}\left\{m_{j i}^{(p)}+v^{p}\right\}+{ }_{j} m_{i i}^{(p)} \leqq 2^{p}\left\{m_{j i}^{(p)}+{ }_{i} m_{i j}^{(p)}\right\}+{ }_{j} m_{i i}^{(p)}
$$

From (4) we obtain, since $(s+v)^{p} \geqq v^{p}$,

$$
m_{i j}^{(p)} \geqq{ }_{j} m_{i i}^{(p)}+{ }_{i} m_{i j}^{(p)} \text {. }
$$

Hence if (1) is true, it follows firstly from (7) that ${ }_{j} m_{i i}^{(p)}<\infty,{ }_{i j} m_{i j}^{(p)}<\infty$, then from (6) that $m_{i 1}^{(p)}<\infty$. By symmetry we have also $m_{j j}^{(p)}<\infty$.

(ii) We shall show that (5) implies (1) for all $j\left(^{5}\right)$. From (3) we obtain, since $(s+v)^{p} \geqq \max \left\{s^{p}, v^{p}\right\}$,

$$
m_{i i}^{(p)} \geqq \max \left\{{ }_{i} F_{i j}^{*} m_{j i}^{(p)},{ }_{i} m_{i j}^{(p)}\right\} .
$$

Since ${ }_{i} F_{i j}^{*}>0$ we have $m_{j i}^{(p)}<\infty$; also ${ }_{i} m_{i j}^{(p)}<\infty$.

Next we have, from (2),

$$
\begin{aligned}
\sum_{n=1}^{N} n{ }^{p} F_{i j}^{(n)}= & \sum_{v=1}^{N-1}{ }_{j} F_{i i}^{(v)} \sum_{s=1}^{N-v} s^{p} F_{i j}^{(s)}+\sum_{v=1}^{N-1}{ }_{j} F_{i i}^{(v)} \sum_{s=1}^{N-v}\left\{(s+v)^{p}-s^{p}\right\} F_{i j}^{(s)} \\
& +\sum_{n=1}^{N} n^{p}{ }_{i} F_{i j}^{(n)} .
\end{aligned}
$$

If $0<p \leqq 1$, then $(s+v)^{p}-s^{p} \leqq v^{p}$. Since ${ }_{j} m_{i i}^{(p)} \leqq m_{i i}^{(p)}<\infty$, and we have just seen that ${ }_{i} m_{i j}^{(p)}<\infty$, it follows on letting $N \rightarrow \infty$ that

$$
\left(1-{ }_{j} F_{i i}^{*}\right) m_{i j}^{(p)} \leqq{ }_{j} m_{i i}^{(p)}+{ }_{i} m_{i j}^{(p)}<\infty .
$$

Since ${ }_{j} F_{i i}^{*}<1$, we have $m_{i j}^{(p)}<\infty$.

If $p>1$, we use the inequality

$$
(s+v)^{p}-s^{p} \leqq p v(s+v)^{p-1} \leqq p 2^{p-1} v\left(s^{p-1}+v^{p-1}\right) .
$$

We thus obtain, similarly to the above,

$$
\left(1-{ }_{j} F_{i i}^{*}\right) m_{i j}^{(p)} \leqq p 2^{p-1}\left\{{ }_{j} m_{i i}^{(1)} m_{i j}^{(p-1)}+{ }_{j} m_{i i}^{(p)}\right\}+{ }_{i} m_{i j}^{(p)} .
$$

Now we are going to use induction on $[p]$. Suppose that we have proved that $m_{i i}^{(p-1)}<\infty$ implies $m_{i j}^{(p-1)}<\infty$ for every $j$. If $m_{i i}^{(p)}<\infty$ then $m_{i i}^{(p-1)}$ $\leqq m_{i i}^{(p)}<\infty$ and so by the induction hypothesis we have $m_{i j}^{(p-1)}<\infty$. Since $p>1$ the assumption $m_{i i}^{(p)}<\infty$ also implies that $m_{i i}^{(1)}<\infty$. Hence it follows from (8) that $m_{i j}^{(p)}<\infty$.

(5) Henceforth $j$ is a general index, not necessarily the specific one in (1). 
Since we have proved that the implication " $m_{i j}^{(p)}<\infty$ implies $m_{i j}^{(p)}<\infty$ " is true for all $p$ with $0<p \leqq 1$, it is now true for all $p>0$, by induction on $[p]$.

(iii) We have thus far proved that if (1) is true for a pair of $i$ and $j$ then it is also true for the same $i$ but all $j$. Hence by (i) also $m_{j j}^{(p)}<\infty$ for all $j$.

REMARK 1. The two conditions in (1) cannot be replaced by one of them. It is possible that $m_{i j}<\infty$ but $m_{j i}=\infty$. Consider for example a random walk with a reflecting barrier: $P_{01}=1, P_{n, n+1}=P_{n, n-1}=1 / 2$ for $n \geqq 1$. This example also shows that some $m_{i j}$ may be finite in a null class.

REMARK 2. Let $\phi(n)$ be a positive, nondecreasing function of $n$ satisfying $\phi\left(n+n^{\prime}\right) \leqq \phi(n)+A \phi\left(n^{\prime}\right)$ for some constant $A>0$ and every pair of positive integers $n$ and $n^{\prime}$ where $A$ is a positive constant. Define a generalized moment $\left.{ }^{6}\right)$ as follows:

$$
m_{i j}^{(\phi)}=\sum_{n=1}^{\infty} \phi(n) F_{i j}^{(n)} .
$$

Then the theorem remains true if we replace $m^{(p)}$ by $m^{(\phi)}$. This is a slight generalization of the theorem for the case $0<p \leqq 1$. The proof is exactly the same as in that case.

REMARK 3. For $p$ a positive integer, we have

$$
\begin{aligned}
m_{i i}^{(p)} & =\sum_{q=0}^{p}\left(\begin{array}{l}
p \\
q
\end{array}\right){ }_{i} m_{i j}^{(p-q)} m_{j i}^{(q)}+{ }_{j} m_{i i}^{(p)}, \\
{ }_{i} F_{i j}^{*} m_{i j}^{(p)} & =\sum_{q=0}^{p-1}\left(\begin{array}{l}
p \\
q
\end{array}\right){ }_{i} m_{i i}^{(p-q)} m_{i j}^{(q)}+{ }_{i} m_{i j}^{(p)} .
\end{aligned}
$$

Kolmogorov [12] proved that if $m_{i i}<\infty$ for some $i$ in a recurrent class, then $m_{j k}<\infty$ for all $j$ and $k$ (distinct or not) in the same class. This is part of the assertion of Theorem 1 for $p=1$. For $p=2$ the result has an important application to Doblin's central limit theorem; see $\$ 4$.

Theorem 1 makes it possible to classify recurrent states and classes. One possibility is as follows. We define the "order" of a recurrent state to be the supremum of all numbers $p \geqq 0$ for which $m_{i i}^{(p)}<\infty$. It follows from Theorem 1 that all the states in one recurrent class have the same order, which may therefore also be called the order of the class. Obviously, a positive state or class (in the established terminology $\left({ }^{7}\right)$ ) is of order $\geqq 1$, and a null state or class is of order $\leqq 1$. A state or class of order 1 may be positive or null, as will appear shortly. We state the following existence theorem.

For any given $p \geqq 0$ there exists a Markov chain such that all of its states form a class of order $p$.

(') I am indebted to J. L. Doob for the suggestion of a generalized moment; unfortunately I do not see how to extend the theorem significantly for all $p$.

(") Lévy [15] uses "strongly ergodic" for "positive" and "weakly ergodic" for "null." 
This follows from a simple lemma $\left(^{8}\right)$ given by Yosida and Kakutani [16], which asserts: Given a sequence of numbers $f_{n}, n \geqq 1$, such that $f_{n} \geqq 0, \sum f_{n}=1$, there exists a Markov chain for which $F_{00}^{(n)}=f_{n}$. To prove the existence theorem above it suffices to take in the lemma

$$
f_{n}=\frac{C}{n^{p+1}} \text { or } \frac{C^{\prime}}{n^{p+1} \lg ^{2} n}
$$

where $p>0$ and $C$ and $C^{\prime}$ are constants such that $\sum_{n} f_{n}=1$. In either case the state 0 is of order $p$; however $m_{00}^{(p)}=\infty$ with the first choice, while $m_{00}^{(p)}<\infty$ with the second. For $p=0$ only the second choice is possible.

2. In this section we consider a positive class and we investigate some relations involving the $m_{i j}$ 's and also give numerical examples.

The following interesting relation is due to T. E. Harris [9]:

$$
\lim _{j \rightarrow \infty} F_{i j}^{*} m_{i j}=m_{i i}
$$

We give a new proof here to put it into closer relationship with our developments. Putting $p=1$ in (9) and (10), we have

$$
\begin{gathered}
m_{i i}={ }_{i} F_{i j}^{*} m_{j i}+{ }_{i} m_{i j}+{ }_{j} m_{i i}, \\
{ }_{i} F_{i j}^{*} m_{i j}={ }_{j} m_{i i}+{ }_{i} m_{i j} .
\end{gathered}
$$

On comparing the two formulas we obtain, firstly,

$$
m_{i i}={ }_{i} F_{i j}^{*}\left(m_{i j}+m_{j i}\right)
$$

also due to Harris ([9]; see (VIIIa) of [1]). Secondly, since $0 \leqq F_{i i}^{(n)}-{ }_{j} F_{i i}^{(n)} \rightarrow 0$ as $j \rightarrow \infty$, the infinite series for ${ }_{j} m_{i i}$ converges uniformly in $j$, and

$$
\lim _{j \rightarrow \infty} m_{i i}=m_{i i}
$$

it follows from $\left(9^{\prime}\right)$ that

$$
\lim _{j \rightarrow \infty} m_{i j}=0, \quad \lim _{j \rightarrow \infty} F_{i j}^{*} m_{j i}=0 .
$$

The last relation and (12) imply (11).

As a simple consequence, we have for every $i$

$$
\sum_{i} \frac{1}{m_{i j}}<\infty .
$$

This follows from the obvious relation $m_{j j} \leqq m_{i j}+m_{j i}$ and Kolmogorov's

(8) It can be easily proved by the scheme in Example 1 below. 
result $\sum_{j=0}^{\infty} 1 / m_{j j}=1$.

For further relations we note the following remarkable formula which expresses the second moment in terms of the first moments:

$$
m_{i i}^{(2)}=m_{i i}\left(2 \sum_{j=0}^{\infty} \frac{m_{j i}}{m_{j i}}-1\right) .
$$

To prove (14) we have only to set $f(\cdot) \equiv 1$ in (B) of the Appendix, and use (C) there:

$$
\begin{aligned}
m_{i i}^{(2)} & =m_{i i}+2 m_{i i} \sum_{j \neq i} \frac{1}{m_{j i}} \sum_{k} \frac{m_{j i}+m_{i k}-m_{j k}}{m_{k k}} \\
& =m_{i i}+2 m_{i i} \sum_{j \neq i} \frac{m_{j i}}{m_{j i}}=m_{i i}\left(2 \sum_{j} \frac{m_{j i}}{m_{j j}}-1\right) .
\end{aligned}
$$

From (14) it follows that the series

$$
\sum_{j} \frac{m_{j i}}{m_{j i}}
$$

converges if and only if $m_{\mathfrak{t}}^{(2)}<\infty$. Consequently, in view of Theorem 1, all such series for different values of $i$ converge or diverge together. If one side of (14) is infinite, so is the other. In particular if $m_{t i}^{(2)}=\infty$, then

$$
\lim _{j \rightarrow \infty} m_{j i}=\infty \text {. }
$$

As a counterpart to the last result we now give an example where $\lim _{j \rightarrow \infty} m_{j 0}=1$. Define a Markov chain as follows:

$$
\begin{aligned}
P_{01} & =1, & & P_{12}=1, \\
P_{n, n+1} & =1 / n^{2}, & & P_{n 0}=1-1 / n^{2}
\end{aligned}
$$

By the Borel-Cantelli lemma the state 0 is recurrent; furthermore $m_{00}=2$ $+m_{20}<\infty$ so that all the states form a positive class. Now

$$
m_{j 0}=1-\frac{1}{j^{2}}+\frac{2}{j^{2}}\left(1-\frac{1}{(j+1)^{2}}\right)+\frac{3}{j^{2}(j+1)^{2}}\left(1-\frac{1}{(j+2)^{2}}\right)+\cdots .
$$

Hence $\lim _{j \rightarrow \infty} m_{j 0}=1$.

We now give another example to illustrate the possible asymptotic behavior of the $m_{i j}$ 's. This example will be used in $\$ 4$ to disprove a statement attributed to Kolmogorov.

EXAMPLE 1. Define a Markov chain as follows: for $n \geqq 0$

$$
P_{n 0}=1-p_{n}, \quad P_{n, n+1}=p_{n} .
$$

Put $p_{0}=1$ and put 


$$
\pi_{0}=1, \pi_{n}=p_{0} p_{1} \cdots p_{n-1}, f_{n}=\pi_{n-1}-\pi_{n} .
$$

According to the lemma of Yosida and Kakutani, we may take

$$
f_{0}=0, \quad f_{n}=\frac{C}{n^{3} \lg ^{2} n} \text { for } n \geqq 2, \quad \sum_{n=1}^{\infty} f_{n}=1 .
$$

Then

$$
\pi_{n}=\sum_{v=n+1}^{\infty} \frac{C}{v^{3} \lg ^{2} v} \sim \frac{C}{2 n^{2} \lg ^{2} n} .
$$

Since $P_{i j}^{(n)}=P_{i, j-1}^{(n-1)} p_{j-1}$ for $0 \leqq i<j$ and we are obviously in a positive class, we have by Kolmogorov's ergodic theorem,

$$
\frac{1}{m_{j j}}=\frac{p_{j-1}}{m_{j-1, j-1}} .
$$

Hence

$$
\frac{1}{m_{i j}}=\frac{\pi_{j}}{m_{00}} \sim \frac{C}{2 m_{00}} \frac{1}{j^{2} \lg ^{2} j} .
$$

On the other hand, by an easy calculation

$$
\begin{aligned}
m_{j 0} & =\left(1-p_{j}\right)+2 p_{j}\left(1-p_{j+1}\right)+3 p_{j} p_{j+1}\left(1-p_{j+2}\right)+\cdots \\
& =\frac{1}{\pi_{j}}\left(f_{j+1}+2 f_{j+2}+3 f_{j+3}+\cdots\right) \sim j .
\end{aligned}
$$

It follows in particular that

$$
\sum_{j} \frac{m_{j 0}}{m_{j j}}<\infty .
$$

This checks with a result above since $m_{00}^{(2)}=\sum_{n=1}^{\infty} n^{2} f_{n}<\infty$.

3 . In this section we study certain sequences of random variables associated with the Markov chain $\left\{X_{n}\right\}, n \geqq 0$. Let $f(\cdot)$ be a real-valued function defined on the non-negative integers and consider the sequence $\left\{f\left(X_{n}\right)\right\}$, $n \geqq 0$. If $f$ has a unique inverse, then the new sequence is also a Markov chain; in general this need not be true. A special case which has been frequently discussed (see $[14$, p. 335] and $[8$, p. 342]) is the case in which $f(\cdot)=\delta_{i}$. for a certain $i$, where $\delta$ is the Kronecker symbol. We shall investigate the asymptotic properties of the partial sums

$$
S_{n}=\sum_{v=0}^{n} f\left(X_{v}\right)
$$

as $n \rightarrow \infty$ and prove some of the classical limit theorems for them. In the 
special case just mentioned these results are easily obtained and one might hope to extend them to the general case by a "linear extension." Such an approach however has not been carried out. Instead we shall use an idea due to Doblin [3] $\left({ }^{9}\right)$, as follows:

Let $i$ be a given state and let $0 \leqq v_{1}<v_{2}<\cdots$ be the successive values of $v$ for which $X_{v}=i$. Let

$$
Y_{s}=\sum_{v=v_{s}+1}^{v_{s+1}} f\left(X_{v}\right) .
$$

The $Y_{s}, s \geqq 1$, are independent random variables with a common distribution, and we have

$$
S_{n}=\sum_{v=0}^{v_{1}} f\left(X_{v}\right)+\sum_{s=1}^{l-1} Y_{s}+\sum_{v=v_{l}+1}^{n} f\left(X_{v}\right) .
$$

Thus the asymptotic properties of $S_{n}$ are closely related to those of $\sum_{s=1}^{l-1} Y_{s}$, whose behavior is classic. It remains to explore this relation at greater length.

We begin with a strong limit theorem which applies to any recurrent class. We state the following lemma.

Lemma. Write ${ }_{i} P_{i j}^{*}=E_{i j}$. Then for every $i, j$, and $k$ we have

$$
E_{i j} E_{j k}=E_{i k} \text {. }
$$

In particular $E_{i i}=1$ and $E_{i j} E_{j i}=1$.

Proof. According to (D) of the Appendix we have

$$
E_{i j}=\lim _{n \rightarrow \infty} \frac{\sum_{v=0}^{n} P_{j i}^{(v)}}{\sum_{j=0}^{n} P_{i i}^{(v)}}
$$

where the limit is finite and not zero. The lemma follows at once.

For a given function $f$ we set

$$
I(f)=\sum_{j=0}^{\infty} E_{i j} f(j) .
$$

It follows from the lemma that the finiteness of $I(|f|)$ is independent of the choice of $i$. Furthermore, if $g$ is another function and if $I(|f|)<\infty, I(|g|)$ $<\infty, I(g) \neq 0$, then the number $I(f) / I(g)$ is independent of $i$.

(9) Here is an instance of an idea, so simple and akin to a familiar one (that of a recurrent event), and yet so new. Doblin himself seemed to have taken some time discovering it; Kolmogorov in [13] gave prominence to it 
Theorem $2\left({ }^{10}\right)$. If $I(|f|)<\infty, I(|g|)<\infty$ and $I(g) \neq 0$, then

$$
P\left\{\lim _{n \rightarrow \infty} \frac{\sum_{v=0}^{n} f\left(X_{v}\right)}{\sum_{v=0}^{n} g\left(X_{v}\right)}=\frac{I(f)}{I(g)}\right\}=1 .
$$

Proof. If $I(|f|)<\infty$, we have evidently (cf. the proof of (A), Appendix),

$$
E\left(Y_{1}\right)=\sum_{n=1}^{\infty} \sum_{j=0}^{\infty}{ }_{i} P_{i j}^{(n)} f(j)=\sum_{j=0}^{\infty}{ }_{i} P_{i j}^{*} f(j)=I(f) .
$$

Let $l=l(n)$ be defined by $v_{l(n)} \leqq n<v_{l(n)+1}$. Suppose first that $f(\cdot)>0$. Let $Y^{\prime}=\sum_{v=0}^{v_{1}} f\left(X_{v}\right)$. Since $v_{1}$ is finite with probability one, regardless of the initial probabilities, so is $Y^{\prime}$. We have from (15)

$$
\sum_{s=1}^{l-1} Y_{s} \leqq \sum_{v=0}^{n} f\left(X_{v}\right) \leqq Y^{\prime}+\sum_{s=1}^{l} Y_{s}
$$

Since the state $i$ is recurrent we have $\lim _{n \rightarrow \infty} l(n)=\infty$ with probability one. By the strong law of large numbers of Kolmogorov and Khintchine, we have

$$
P\left\{\lim _{n \rightarrow \infty} \frac{1}{l(n)} \sum_{s=1}^{l(n)} Y_{s}=E\left(Y_{1}\right)\right\}=1 .
$$

It follows from this and (18) that

$$
P\left\{\lim _{n \rightarrow \infty} \frac{1}{l(n)} \sum_{v=0}^{n} f\left(X_{v}\right)=I(f)\right\}=1 .
$$

The same result holds if $f(\cdot)<0$. Hence it holds in general if $I(|f|)<\infty$.

Replacing $f$ by $g$ in (19) and combining the two results we obtain the theorem.

Theorem 2 may be regarded as an ergodic theorem in the form given by E. Hopf $[11$, p. 47$]$. An interesting feature is its validity even though there may be no stationary absolute probabilities for the Markov chain( $\left.{ }^{11}\right)$. Such probabilities are given by the ergodic limits $P_{j}=\lim _{n \rightarrow \infty} d^{-1} P_{j j}^{(n d)}, j \geqq 0$, where $d$ is the period of the class, provided that they are all positive-in other words, if we are in a positive class. Theorem 2 asserts that in any recurrent class, positive or null, we may take instead of the $P_{j}$ the numbers $E_{i j}={ }_{i} P_{i j}^{*}$, $j \geqq 0$, for any choice of $i$. Note that $\sum_{j=0}^{\infty} E_{i j}$ converges if and only if the class

${ }^{(10)}$ After this paper was finished I was informed that T. E. Harris and H. E. Robbins [17] obtained a more general formulation by means of ergodic theory. In our (discrete space) case metric transitivity can also be proved by martingale theory, as pointed out by J. L. Marty. The present proof remains the simplest in this special case.

(11) For an elucidation of the matter see [17]. 
is positive and then $E_{i j}=m_{i i} / m_{j j}$ (see (7) of the Appendix). In this case the theorem specializes to Theorem 6 below, and becomes also a consequence of Birkhoff's theorem (metric transitivity can be proved by Theorem 1.1, p. 460 of [6]). These numbers $E_{i j}$ possess another property of the stationary absolute probabilities $P_{j}$, namely, they satisfy the familiar system of equations

$$
u_{k}=\sum_{j=0}^{\infty} u_{j} P_{j k}
$$

The proof $\left({ }^{12}\right)$ is immediate since (noting that ${ }_{i} P_{i t}^{n}=F_{i i}^{n}$ )

$$
\begin{aligned}
\sum_{j=0}^{\infty}{ }_{i} P_{i j}^{*} P_{j k} & =\sum_{n=1}^{\infty} \sum_{j=0}^{\infty}{ }_{i} P_{i j}^{(n)} P_{j k}=\sum_{n=1}^{\infty}\left\{{ }_{i} P_{i k}^{(n+1)}+{ }_{i} P_{i i}^{(n)} P_{i k}\right\} \\
= & \sum_{n=2}^{\infty}{ }_{i} P_{i k}^{(n)}+P_{i k}={ }_{i} P_{i k .}^{*} .
\end{aligned}
$$

In fact, Derman also proved that the $E_{i j}$, except for a constant of proportionality, are the only solutions of the system (20) which are all positive.

Corollary 1. Let $N_{j}(n)$ and $N_{k}(n)$ be the number of $v^{\prime} s, 0 \leqq v \leqq n$, for which $X_{v}=j$ and $=k$ respectively, then

$$
P\left\{\lim _{n \rightarrow \infty} \frac{N_{j}(n)}{N_{k}(n)}=\frac{E_{i j}}{E_{i k}}\right\}=1 .
$$

This was proved by Harris [9] and Lévy [14], independently. It is the special case of Theorem 2 with $f(\cdot)=\delta_{j}$. and $g(\cdot)=\delta_{k}$, where $\delta$ is the Kronecker symbol.

Corollary 2. Suppose $X_{n}=X_{0}+\xi_{1}+\cdots+\xi_{n}, n \geqq 0$, where $X_{0}$ and the $\xi$ 's are independent, integral-valued random variables and where the $\xi$ 's have a common distribution such that every integer is a recurrent value for the sequence $\left\{X_{n}\right\}, n \geqq 0$; the last condition is satisfied if $E\left(\xi_{1}\right)=0$ (see [2]). If $f$ and $g$ are real-valued functions on the integers such that

then

$$
\sum_{j=-\infty}^{\infty}|f(j)|<\infty, \quad \sum_{j=-\infty}^{\infty}|g(j)|<\infty, \quad \sum_{j=-\infty}^{\infty} g(j) \neq 0
$$

$$
P\left\{\lim _{n \rightarrow \infty} \frac{\sum_{v=1}^{n} f\left(X_{v}\right)}{\sum_{v=1}^{n} g\left(X_{v}\right)}=\frac{\sum_{j=-\infty}^{\infty} f(j)}{\sum_{j=-\infty}^{\infty} g(j)}\right\}=1 .
$$

To prove the corollary we need only note that this is the special case

(12) A longer proof was first given by C. Derman, in his doctoral thesis (Columbia 1954). 
where $P_{i j}$ is a function of $i-j$ and where all $E_{i j}=1$. (Here the states are all the integers instead of the non-negative ones; only a notational change is called for.) This fact, remarkable in its probability interpretation( $\left.{ }^{13}\right)$, follows at once from (16) since $P_{i l}^{(v)}=P_{j j}^{(v)}$ for all $i, j$ and $v$.

4. We now consider a positive recurrent class and use the same notations as in $\S 3$. If the mean of $Y_{s}$ exists $\left({ }^{14}\right)$ we denote it by

$$
E\left(Y_{s}\right)=\mu_{i} \text {. }
$$

In particular if we take $f(\cdot) \equiv 1$ then $Y_{s}$ reduces to the $s$ th recurrence time for the state $i$. We denote this by

$$
T_{s}=v_{s+1}-v_{s}, \quad s \geqq 1,
$$

and we have $E\left(T_{\mathrm{s}}\right)=m_{i i}$. Let

$$
Z_{s}=Y_{s}-\left(\mu_{i} / m_{i i}\right) T_{s} .
$$

The $Z_{s}$ 's are independent random variables with a common distribution whose mean is

$$
E\left(Z_{s}\right)=\mu_{i}-\frac{\mu_{i}}{m_{i i}} m_{i i}=0,
$$

provided that $\mu_{i}$ exists. We note that

$$
Z_{s}=\sum_{v=v_{s}+1}^{v_{s}+1} g\left(X_{v}\right)
$$

where

$$
g(\cdot)=f(\cdot)-\mu_{i} / m_{i i} \cdot
$$

The variance of $Z_{s}$, finite or not, is denoted by

$$
0 \leqq E\left(Z_{s}^{2}\right)=\sigma_{i}^{2} \leqq \infty .
$$

It should be noted that $\sigma_{i}^{2}$ is not the variance of $Y_{s}$.

We first prove a preliminary result which may be regarded as an extension of Theorem 1.

THEOREM 3. Either all $\mu_{i}, i \geqq 0$, exist or none exists. Either all $\sigma_{i}^{2}, i \geqq 0$, are finite or they are all infinite $\left({ }^{15}\right)$.

Proof. Let the $v_{s}$ be defined as above. Let $j \neq 1$ and let $\tau$ be the smallest

${ }^{(13)}$ It states that in any recurrent random walk (with independent, stationary steps) on the integers, the expected number of stops at $j$ between two consecutive stops at $i$ is equal to one for all $i$ and $j$.

(14) A moment exists if it exists and is finite.

(15) Theorem 3 can be extended to absolute moments of all orders $p>0$. 
$n>v_{1}$ such that $X_{n}=j, \tau^{\prime}$ the smallest $n>\tau$ such that $X_{n}=j$, and $v_{N+1}$ be the smallest $n>\tau^{\prime}$ such that $X_{n}=i$.

Furthermore, let $N^{\prime}$ and $N^{\prime \prime}$ be two random variables defined as follows: $N^{\prime}$ is the smallest $s \geqq 1$ such that $X_{n}=j$ for at least one $n, v_{s}<n \leqq v_{s+1}$; and $N^{\prime \prime}$ is the smallest $s \geqq 1$ such that $X_{n}=j$ for at least one $n, v_{N^{\prime}+8}<n \leqq v_{N^{\prime}+8+1}$. Then $N^{\prime}$ and $N^{\prime \prime}$ have the same distribution and $E\left(N^{\prime}\right)=\sum_{n=1}^{\infty} n\left({ }_{j} F_{i i}^{*}\right)^{n-1}{ }_{i} F_{i j}^{*}$ $=1 /{ }_{i} F_{i}^{*}<\infty$.

It is clear that $N \leqq N^{\prime}+N^{\prime \prime}$ so that $E(N) \leqq 2 E\left(N^{\prime}\right)<\infty$.

Consider the $\operatorname{sum}\left({ }^{16}\right)$

$$
W=Z_{1}+\cdots+Z_{N}
$$

We have

$$
E(|W|) \leqq E\left(\sum_{s=1}^{N}\left|Z_{s}\right|\right) \leqq E\left(\sum_{s=1}^{N^{\prime}+N^{\prime \prime}}\left|Z_{s}\right|\right)=2 E\left(\sum_{s=1}^{N^{\prime}}\left|Z_{s}\right|\right) .
$$

If $\mu_{i}$ exists, each $E\left(\left|Z_{\boldsymbol{s}}\right|\right)<\infty$; hence also the conditional expectations

$$
\begin{aligned}
& E_{1}=E\left\{\left|Z_{s}\right| \mid X_{n} \neq j \text { for all } n, v_{s}<n \leqq v_{s+1}\right\}, \\
& E_{2}=E\left\{\left|Z_{s}\right| \mid X_{n}=j \text { for at least one } n, v_{s}<n \leqq v_{s+1}\right\}
\end{aligned}
$$

are both finite. It follows that

$$
\begin{aligned}
E(|W|) & \leqq 2\left(\sum_{s=1}^{N^{\prime}}\left|Z_{s}\right|\right)=2 \sum_{n=1}^{\infty} P\left(N^{\prime}=n\right) E\left\{\sum_{s=1}^{N^{\prime}}\left|Z_{s}\right| \mid N^{\prime}=n\right\} \\
& =2 \sum_{n=1}^{\infty} P\left(N^{\prime}=n\right)\left\{(n-1) E_{1}+E_{2}\right\} \\
& =2\left(E\left(N^{\prime}\right)-1\right) E_{1}+2 E_{2}<\infty .
\end{aligned}
$$

On the other hand,

$$
\begin{aligned}
W & =\sum_{n=v_{1}+1}^{\tau} g\left(X_{n}\right)+\sum_{n=\tau+1}^{\tau^{\prime}} g\left(X_{n}\right)+\sum_{n=\tau^{\prime}+1}^{v_{N+1}} g\left(X_{n}\right) \\
& =W_{1}+W_{2}+W_{3}, \text { say. }
\end{aligned}
$$

The three random variables $W_{1}, W_{2}$, and $W_{3}$ are independent. This is a slight generalization of Doblin's idea mentioned at the beginning of $\S 3$. A formal verification lies in the observation that the "elementary" probabilities concerned:

$$
\begin{aligned}
& v_{1}=a ; \quad \tau=t ; \quad X_{n}=i_{n} \quad \text { for } \quad a+1 \leqq n \leqq t ; \\
& \tau=t ; \quad \tau^{\prime}=t^{\prime} ; \quad X_{n}=i_{n} \text { for } t+1 \leqq n \leqq t^{\prime} ; \\
& \tau^{\prime}=t^{\prime} ; \quad v_{N+1}=b ; \quad X_{n}=i_{n} \quad \text { for } t^{\prime}+1 \leqq n \leqq b
\end{aligned}
$$

${ }^{(16)}$ This idea is suggested by J. Wolfowitz in connection with another proof of Theorem 1. 
where $a<t<t^{\prime}<b$, are in fact multiplicative. Furthermore $E\left(W_{1}\right)=E\left(W_{2}\right)$ $=E\left(W_{3}\right)=0$. Hence applying an inequality due to Doob [5, Lemma 2], we have by (21)

$$
E\left(\left|W_{2}\right|\right) \leqq E(|W|)<\infty .
$$

By the definition of $\tau$ and $\tau^{\prime}$ it is clear that $\mu_{j}=E\left(W_{2}\right)+\left(\mu_{i} / m_{i i}\right) m_{j j}$. Since $j$ is arbitrary we have thus proved that the existence of any $\mu_{i}$ implies that of all $\mu_{i}$.

Similarly, by considering the variances and using the additivity of the variances of independent random variables we see that the finiteness of any $\sigma_{i}^{2}$ implies that of all $\sigma_{i}^{2}$.

REMARK. If we set $f(\cdot) \equiv 1$ in Theorem 3 , the results reduce to part of the assertion of Theorem 1 for $p=1$ and $p=2$.

We are now in a position to prove the classical limit theorems for the sequence $\left\{f\left(X_{n}\right)\right\}$. Note that, thanks to Theorem 3 , the hypotheses in the following theorems are invariant if the state $i$ is replaced by any other state. In the following we shall write $\mu_{i} / m_{i i}$ as $M$, and $\sigma_{i}^{2} / m_{i i}$ as $B$. It will turn out presently that these numbers, if they exist, are in fact independent of the choice of $i$, thus justifying the notations.

TheOREM 4 (WEAK LAW OF LARGE NUMBers). If $m_{i i}<\infty$ and $\mu_{i}$ exists, then for every $\epsilon>0$,

$$
\lim _{n \rightarrow \infty} P\left\{\left|\frac{S_{n}}{n}-M\right|>\epsilon\right\}=0 .
$$

Proof. Let the $v_{s}$ and $T_{s}$ be as before. For every $n \geqq 0$ define $l=l(n)$ by

$$
v_{\imath} \leqq n<v_{l+1} .
$$

We have then

$$
S_{n}-\sum_{v=1}^{l-1} M T_{v}=Y^{\prime}+\sum_{v=1}^{l-1} Z_{v}+Y^{\prime \prime}
$$

where

$$
Y^{\prime}=\sum_{v=0}^{v_{1}} f\left(X_{v}\right), \quad Y^{\prime \prime}=\sum_{v=v_{l}+1}^{n} f\left(X_{v}\right) .
$$

Since $v_{1}$ is finite with probability one, it is clear that $\lim _{u \rightarrow \infty} P\left(\left|Y^{\prime}\right|>u\right)=0$. However $Y^{\prime \prime}=Y_{n}^{\prime \prime}$ depends on $n$. To show that $\lim _{u \rightarrow \infty} P\left(\left|Y_{n}^{\prime \prime}\right|>u\right)=0$ uniformly with respect to $n$ we need a simple result due to Kolmogorov [12] according to which

$$
\lim _{t \rightarrow \infty} P\left(n-v_{l(n)}>t\right)=0
$$


uniformly with respect to $n$. It follows that

$$
P\left(\left|Y^{\prime \prime}\right|>u\right) \leqq P\left(\max _{1 \leqq s \leqq t}\left|\sum_{v=v_{l}+1}^{v_{l}+s} f\left(X_{v}\right)\right|>u\right)+P\left(n-v_{l}>t\right) .
$$

Hence the left side tends to 0 uniformly in $n$.

Applying Khintchine's weak law of large numbers to the sequence $\left\{Z_{s}\right\}$, $s \geqq 1$, we obtain

$$
\lim _{n \rightarrow \infty} P\left(\left|S_{n}-M \sum_{v=1}^{l-1} T_{v}\right|>n \epsilon\right)=0,
$$

since $\sum_{v=1}^{l-1} T_{v}=v_{l}$ and $P\left(\lim _{n \rightarrow \infty} v_{l(n)} / n=1\right)=1$. This is equivalent to (22).

COROLlary. Under the hypotheses of the theorem all $\mu_{j} / m_{j j}, 0 \leqq j<\infty$, are equal.

TheOREM 5. (Doblin's CENTRAL Limit THEOREM). If $m_{i i}^{(2)}<\infty$ and $0<\sigma_{i}^{2}$ $<\infty\left({ }^{17}\right)$, then for every real $x$

$$
\lim _{n \rightarrow \infty} P\left\{\frac{S_{n}-M n}{(B n)^{1 / 2}} \leqq x\right\}=\frac{1}{(2 \pi)^{1 / 2}} \int_{-\infty}^{x} e^{-y^{2 / 2}} d y .
$$

The proof of Theorem 5 is completely similar to that of Theorem 4 if we apply the central limit theorem for independent, identically distributed random variables with finite variances. We remark that this proof is considerably simpler in details than the one given originally by Doblin [3], who made several unnecessary estimates. Doblin also proved that if $\sigma_{i}^{2}=0, S_{n}$ is the sum of $M n$ and a constant depending only on the values of $X_{0}$ and $X_{n}$.

CoRollaRy 1. Under the hypotheses of the theorem all $\sigma_{j}^{2} / m_{j j}, 0 \leqq j<\infty$, are equal.

CoRollary 2. If $f(\cdot)$ is a bounded function, then Theorem 4 holds under the sole assumption that $m_{i i}<\infty$, and Theorem 5 holds under the sole assumption that $m_{i t}^{(2)}<\infty$ if we allow a degenerate normal distribution in case $\sigma_{i}^{2}=0$. In particular, the theorems hold if the number of states is finite without any assumption whatsoever (in a class of mutually communicating states).

Corollary 3. The constants $M$ and $B$ in Theorems 4 and 5 are given by

$$
\begin{gathered}
M=\sum_{j=0}^{\infty} \frac{f(j)}{m_{j j}}, \\
B=\sum_{j=0}^{\infty} \frac{g^{2}(j)}{m_{j j}}+2 \sum_{j=0, j \neq i}^{\infty} \frac{g(j)}{m_{j j}} \sum_{k=0}^{\infty} \frac{m_{j i}+m_{i k}-m_{j k}}{m_{k k}} g(k)
\end{gathered}
$$

(17) Under the assumption $m_{i \imath}^{(2)}<\infty$ the condition $\sigma_{i}^{2}<\infty$ is obviously equivalent to $E\left(Y_{1}^{2}\right)<\infty$. 
provided that the series converge absolutely.

This follows from (A) and (B) of the Appendix. It is easy to see that $M$ may exist without the series on the right side of (24) being absolutely convergent. We give the following example which will be used also in $\$ 5$.

EXAmple 2. Define a Markov chain $\left({ }^{18}\right)$ as follows:

$$
P_{0,2 i-1}=\frac{6}{\pi^{2} i^{2}}, \quad P_{2 i i-1,2}=1, \quad P_{2 i, 0}=1, \quad i \geqq 1 .
$$

Let $f(0)=0, f(2 i-1)=i, f(2 i)=-i$ for $i \geqq 1$. Obviously $m_{00}=3$ and $m_{00}^{(2)}=9$. It is easy to see that $m_{i i}=\pi^{2} i^{2} / 2$ for $i \geqq 1$. Hence $\sum_{j}|f(j)| / m_{j j}=\infty$. On the other hand, both $M$ and $B$ are equal to zero.

According to Doblin [3], Kolmogorov stated in a letter to Fréchet in 1937 that the central limit theorem applies to $S_{n}$ under the following conditions:

$$
m_{i i}^{(2)}<\infty, \quad \sum_{j=0}^{\infty} \frac{f^{2}(j)}{m_{j j}}<\infty .
$$

Apart from this indirect reference this statement has never been published to our knowledge $\left({ }^{19}\right)$. We think that it is false, as will be shown by the following example $\left({ }^{20}\right)$.

Example 3. Consider Example 1 and set

$$
f(j)=m_{j 0}^{1 / 2},
$$

Then $m_{00}^{(2)}<\infty$ as shown there and

$$
\sum_{j} \frac{f^{2}(j)}{m_{j j}}=\sum \frac{m_{j 0}}{m_{j j}}<\infty,
$$

so that (26) is satisfied.

We now rewrite (23) in the form

$$
S_{n}=Y^{\prime}+\sum_{v=1}^{l-1} Y_{v}+Y^{\prime \prime}
$$

It is clear that if the central limit theorem holds for $S_{n}$, it would also hold for the sequence of (partial sums of) the independent, identically distributed

(18) This chain is periodic with period 3. A trivial modification makes it nonperiodic.

${ }^{(19)}$ In a subsequent paper [13] on the local central limit theorem Kolmogorov treated only the case of a finite number of states and used Doblin's method.

${ }^{(20)}$ Added in proof. Even if the second condition in (26) is strengthened to $\sum f^{r}(j) / m_{j i}<\infty$ for an $r>2$ it is still not sufficient. This can be shown by setting $f(j)=j^{1 / r}$ and $f_{n}=C n^{-3}(\lg n)^{-2}$ in Example 3. Cf. a theorem of Doblin-Doob [6, Theorem 7.5, p. 228]. 
random variables $Y_{s}, s \geqq 1$. Write $Y_{1}=Y$. By the construction of the chain in Example 1 we have

$$
P\left(Y=m_{00}^{1 / 2}+m_{10}^{1 / 2}+\cdots+m_{n-1,0}^{1 / 2}\right)=f_{n}=\frac{C}{n^{3} \lg ^{2} n}, \quad n \geqq 2 .
$$

Since $m_{j 0} \sim j$ it follows that $\sum_{j=0}^{n-1} m_{j 0}^{1 / 2} \sim 2 n^{3 / 2} / 3$. As $x \rightarrow \infty$, we have

$$
P(Y>x) \sim \sum_{2 n^{3 / 2 / 3>x}} f_{n}=\sum_{n>(3 x / 2)^{2 / 3}} \frac{C}{n^{3} \lg ^{2} n} \sim \frac{C_{1}}{x^{4 / 3} \lg ^{2} x} .
$$

On the other hand, we have

$$
\int_{0}^{x} y^{2} d_{y} P(Y \leqq y) \sim \sum_{2 n^{3 / 2} / 3 \leqq x}\left(\frac{2}{3} n^{3 / 2}\right)^{2} f_{n}=C_{2} \sum_{n \leqq(3 x / 2)^{2 / 3}} \frac{n^{3}}{n^{3} \lg ^{2} n} \sim C_{3} \frac{x^{2 / 3}}{\lg ^{2} x}
$$

where $C_{1}, C_{2}$, and $C_{3}$ are positive constants. Therefore,

$$
\lim _{x \rightarrow \infty} \frac{x^{2} P(Y>x)}{\int_{0}^{x} y^{2} d_{y} P(Y \leqq y)}=\frac{C_{1}}{C_{3}}>0 .
$$

According to the necessary and sufficient condition ( $\left.{ }^{21}\right)$ of Feller and Lévy for the identically distributed case $[15$, p. 113] this proves that the central limit theorem does not hold for the sequence $\left\{Y_{s}\right\}$; hence it does not hold for the sequence $S_{n}$. In particular, Doblin's condition $\sigma_{i}^{2}<\infty$ is not satisfied; in fact as shown above

$$
\int_{0}^{\infty} y^{2} d_{y} P(Y \leqq y)=\infty .
$$

On the other hand, Example 2 shows that Doblin's conditions $m_{i t}^{(2)}<\infty$ and $\sigma_{i}^{2}<\infty$ do not imply (26).

5. The conditions that $m_{i i}<\infty$ and $\mu_{i}$ exists, sufficient for the weak law of large numbers, are not sufficient for the strong law. To see that, we need only return to Example 2. Suppose $X_{0}=0$ with probability one. If $X_{v}=0$ for a certain $v$, then the probability that $f\left(X_{v+1}\right) \geqq n$ is equal to

$$
\frac{6}{\pi^{2}} \sum_{i=n}^{\infty} \frac{1}{i^{2}} \sim \frac{6}{\pi^{2} n} .
$$

Since $S_{3 n}=0$ for every $n \geqq 0$, we have then $S_{3 n+1} \geqq n$ with the above probability. It follows by the Borel-Cantelli lemma that $S_{3 n+1} \geqq n$ infinitely of ten with

${ }^{(21)}$ Clearly we can state necessary and sufficient conditions for some of the limit theorems given here if we use the distribution function of $Y$. To our mind such statements are absolutely futile. 
probability one. Hence the strong law of large numbers does not hold, while the weak law holds trivially.

This example suggests that the sufficient condition given in the next theorem, though not necessary $\left({ }^{22}\right)$, is probably not very far from the truth.

Theorem 6 (Strong LAW OF LARGE NUMBERS). If $m_{i i}<\infty$ and

$$
\sum_{j=0}^{\infty} \frac{|f(j)|}{m_{j i}}<\infty
$$

then

$$
P\left\{\lim _{n \rightarrow \infty} \frac{S_{n}}{n}=M\right\}=1 .
$$

REMARK. If the chain $X_{n}$ is stationary, then under (27) the number $M$ is simply $E\left(f\left(X_{0}\right)\right)$.

Proof. The proof of this theorem is implicit in that of Theorem 2; it is also a corollary of that theorem for $g(\cdot) \equiv 1$. We need only note that since we are now in a positive class,

$$
E_{i j}=m_{i i} / m_{j j}
$$

and $P\left(\lim _{n \rightarrow \infty} l(n) / n=1 / m_{i i}\right)=1$.

The following alternative method seems instructive. Referring back to the proof of Theorem 4, we see that we may apply the strong law of large numbers of Kolmogorov and Khintchine to $\left\{Z_{s}\right\}$. It remains thus to prove that

$$
P\left(\lim _{n \rightarrow \infty} \frac{Y_{n}^{\prime \prime}}{n}=0\right)=1 \text {. }
$$

A little reflection shows that this is equivalent to

$$
P\left\{\max _{v_{l}<v^{\prime} \leqq v_{l+1}}\left|\sum_{v=v_{l}+1}^{v^{\prime}} f\left(X_{v}\right)\right|>n \in \text { i.o. }\right\}=0 .
$$

Let $U_{l}=\sum_{v=v_{l}+1}^{v_{l} l}\left|f\left(X_{v}\right)\right|$, then (27) is equivalent to $E\left(U_{l}\right)<\infty$. Since the $U_{l}, l \geqq 1$, have a common distribution it follows by a well known inequality that

$$
\sum_{n} P\left(U_{l(n)}>n \epsilon\right)<\infty .
$$

Hence by the Borel-Cantelli lemma,

$$
P\left(U_{l(n)}>n \epsilon \text { i.o. }\right)=0 .
$$

This obviously implies (28) and proves the theorem.

(22) A slight modification of Example 2 confirms this. 
It is interesting that a similar strengthening of the conditions of Theorem 5, obtained by substituting $|f|$ for $f$, leads to the law of the iterated logarithm.

Theorem 7 (LAw of the iterated LOGarithm) $\left({ }^{23}\right)$. If $m_{i \imath}^{(2)}<\infty$, and if

$$
E\left\{\left[\sum_{v=v_{1}+1}^{v_{2}}\left|f\left(X_{v}\right)\right|\right]^{2}\right\}<\infty
$$

and $B>0$, then

$$
P\left\{\limsup _{n \rightarrow \infty} \frac{S_{n}-M n}{(B n \lg \lg n)^{1 / 2}}=1\right\}=1 .
$$

REMARK. Using the random variables $U_{l}$ introduced in the proof of Theorem 6, the left-hand side of (29) may be written as $E\left(U_{1}^{2}\right)$. Using (B) of the Appendix it may also be written as the right-hand side of (B) after substituting $|f|$ for $f$.

Proof. As before we may ignore $Y^{\prime}$ in (23). According to the law of the iterated logarithm in the form given by Hartman and Wintner [10], we have

$$
P\left\{\limsup _{l \rightarrow \infty} \frac{\sum_{v=1}^{l-1} Z_{v}}{\left(l \sigma_{i}^{2} \lg \lg l\right)^{1 / 2}}=1\right\}=1 .
$$

Since $P\left(\lim _{n \rightarrow \infty} l(n) / n=1 / m_{i i}\right)=1$ the limit may be taken as $n \rightarrow \infty$ and the denominator may be replaced by $(B n \lg \lg n)^{1 / 2}$.

It remains to prove, as in the previous proof, that for every $\epsilon>0$

$$
P\left\{U_{l(n)}>(n \lg \lg n)^{1 / 2} \epsilon \text { i.o. }\right\}=0 .
$$

Now it is easy to see that

$$
\sum_{n} P\left(U_{l(n)}>n^{1 / 2}\right) \leqq C E\left(U_{1}^{2}\right)<\infty
$$

where $C$ is some positive constant. Hence we have as before

$$
P\left(U_{l(n)}>n^{1 / 2} \text { i.o. }\right)=0 .
$$

This is more than we need to prove the theorem.

Finally, we prove the limit theorems concerning $\max _{0 \leqq v \leqq n} S_{v}$ and $\max _{0 \leqq v \leqq n}\left|S_{v}\right|$ respectively.

THEOREM $\left.8{ }^{24}\right)$. Under the same hypotheses as in Theorem 7, we have

${ }^{(23)}$ For the case of a finite number of states see Doblin [3], where a sharper result is proved by a longer method.

(24) The limit joint distribution of $\max \left(S_{v}-M v\right)$ and $\min \left(S_{v}-M v\right)$ can be similarly derived. 


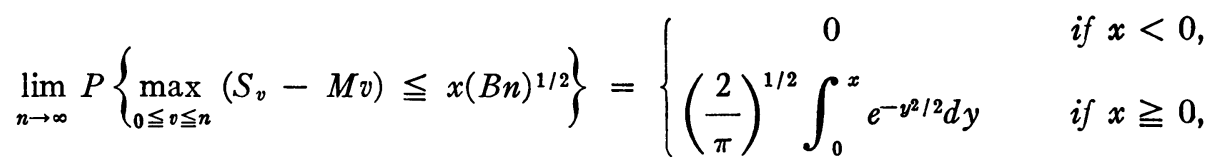$$
\lim _{n \rightarrow \infty} P\left\{\max _{0 \leqq v \leqq n}\left|S_{v}-M v\right| \leqq x(B n)^{1 / 2}\right\}
$$$$
=\frac{4}{\pi} \sum_{m=0}^{\infty} \frac{(-1)^{m}}{2 m+1} \exp \left\{\frac{-(2 m+1)^{2} \pi^{2}}{8 x^{2}}\right\} \quad \text { if } x \geqq 0 .
$$

Proof. We shall be brief now. According to results of Erdös and Kac [17], these limit theorems hold for the sequence of random variables $\left\{Z_{n}\right\}, n \geqq 1$. Hence it is sufficient to show that for every $\epsilon>0$

$$
\begin{array}{r}
\lim _{n \rightarrow \infty} P\left\{\max _{1 \leqq s_{s}<l} \max _{v_{s}<t \leqq v_{s+1}}\left|\sum_{v=v_{v}+1}^{t} f\left(X_{v}\right)\right|>n^{1 / 2} \epsilon\right\}=0, \\
\lim _{n \rightarrow \infty} P\left\{\max _{a \leqq t \leqq v_{1}}\left|\sum_{v=1}^{t} f\left(X_{v}\right)\right|>n^{1 / 2} \epsilon\right\}=0, \\
\lim _{n \rightarrow \infty} P\left\{\max _{v_{l}<t \leqq n}\left|\sum_{v=v_{l}+1}^{t} f\left(X_{v}\right)\right|>n^{1 / 2} \epsilon\right\}=0 .
\end{array}
$$

We need only consider the first relation, the other two being implicit in the proof of Theorem 4 . The probability written there is clearly not greater than

$$
\begin{aligned}
n P\left\{\sum_{v=v_{1}+1}^{v_{2}}\left|f\left(X_{v}\right)\right|>n^{1 / 2} \epsilon\right\} & =n P\left(U_{1}>n^{1 / 2} \epsilon\right) \\
& \leqq n \frac{1}{n \epsilon^{2}} \int_{U_{1}>n^{1 / 2} \epsilon} U_{1} d P r=\frac{1}{\epsilon^{2}} \int_{U_{1}>n^{1 / 2} \epsilon} U_{1} d P r
\end{aligned}
$$

which clearly tends to zero as $n \rightarrow \infty$. This proves the theorem.

We forbear from stating further limit theorems which can be proved by the same method, but add a word about the conditions (27) and (29) which appear in Theorems 6,7 , and 8 . We note that they are clearly satisfied if $m_{i t}^{(2)}<\infty$ and if $f$ is a bounded function. In particular, Theorems 6,7 , and 8 hold without any assumption (in a class of mutually communicating states) if the number of states is finite. We note furthermore that if we assume only the weaker condition that $\mu_{i}$ exists (in Theorem 6) or $\sigma_{i}^{2}<\infty$ (in Theorems 7 and 8 ), then we need an inequality of the following form: for some constant $c>0$ and every $x>0$,

$$
P\left\{\max _{v_{1}<t \leqq 0_{2}}\left|\sum_{v=v_{1}+1}^{t} f\left(X_{v}\right)\right|>x\right\} \leqq c P\left\{\left|\sum_{v=v_{1}+1}^{v_{2}} f\left(X_{v}\right)\right|>x\right\} .
$$

It is not known what reasonable process, if any, enjoys this property, but 
it is somewhat reminiscent of martingales. At least in one case, namely Theorem 8 , the condition (29) may be replaced by the weaker one $\sigma_{i}^{2}<\infty$ and the additional assumption that $S_{n}=\sum_{v=0}^{n} f\left(X_{v}\right), n \geqq 0$, is a martingale. This means that the following equations are satisfied for all $i \geqq 0$ :

$$
\sum_{j=0}^{\infty} P_{i j} f(j)=f(i), \quad \sum_{j=0}^{\infty} P_{i j}|f(j)|<\infty .
$$

Then the sequence $V_{n}=\sum_{v=v_{1}+1}^{v_{1}+n} f\left(X_{v}\right), n \geqq 1$, is a martingale and the process obtained from $V_{n}$ by optional stopping:

$$
V_{1}, \cdots, V_{v_{2}-v_{1}}
$$

is also a martingale (see $\left[6\right.$, Theorem 2.1, p. 300]). The process $V_{n}^{2}, 1 \leqq n$ $\leqq v_{2}-v_{1}$, is then a semi-martingale (loc. cit., Theorem 1.1, p. 295) and we have (loc. cit., Theorem 3.2, p. 314)

$$
n P\left\{\max _{1 \leqq t \leqq v_{2}-v_{1}}\left|V_{t}\right|>n^{1 / 2} \epsilon\right\} \leqq \frac{1}{\epsilon^{2}} \int_{\max _{1} \leqq t \leqq v_{2}-v_{1}\left|V_{t}\right|>n^{1 / 2} \epsilon} V_{v_{2}-v_{1}}^{2} d P r .
$$

The conditions $m_{t i}^{(2)}<\infty$ and $\sigma_{i}<\infty$ imply that $E\left(V_{v_{2}-v_{1}}^{2}\right)<\infty$. Since $\max _{1 \leqq t \leqq v_{2}-v_{1}}\left|V_{t}\right|$ is finite with probability one, the right side of (31) tends to 0 as $n \rightarrow \infty$, and this is sufficient to prove the first relation in (30).

\section{APPENDIX}

For the sake of convenience we state and prove some results from [1] which are used in the present paper.

In the notations used at the beginning of $\S 3$, we have (Theorem 3 of [1]):

$$
\begin{aligned}
E\left\{\sum_{v=v_{1}+1}^{v_{2}} f\left(X_{v}\right)\right\}= & m_{i i} \sum_{j=0}^{\infty} \frac{f(j)}{m_{j i}}, \\
E\left\{\left[\sum_{v=v_{1}+1}^{v_{2}} f\left(X_{v}\right)\right]^{2}\right\}= & m_{i i} \sum_{j=0}^{\infty} \frac{f^{2}(j)}{m_{j i}} \\
& +2 m_{i i} \sum_{j=0, j \neq i}^{\infty} \frac{f(j)}{m_{j i}} \sum_{k=0}^{\infty} \frac{m_{j i}+m_{i k}-m_{j k}}{m_{k k}} f(k),
\end{aligned}
$$

provided that the series converge absolutely. Furthermore (see (19) of [1]),

$$
\sum_{k=0}^{\infty} \frac{m_{j i}+m_{i k}-m_{j k}}{m_{k k}}=m_{j i}
$$

Proof. If $j \neq k$ we have

$$
F_{j k}^{(n)}=\sum_{v=0}^{n-1}{ }_{k} P_{j j}^{(v)}{ }_{j} F_{j k}^{(n-v)}
$$


where ${ }_{k} P_{j j}^{(0)}=1$. Summing over $n$ we obtain

$$
1=F_{j k}^{*}={ }_{j} F_{j k}^{*}\left(1+{ }_{k} P_{j i}^{*}\right) \text {. }
$$

Further, if $j \neq k$ we have ${ }_{k} P_{i j}^{(n)}=\sum_{0=1}^{n}{ }_{k} F_{k j}^{(0)}{ }_{k} P_{j j}^{(n-v)}$. Summing over $n$ we obtain

$$
{ }_{k} P_{i j}^{*}={ }_{k} F_{i j}^{*}\left(1+{ }_{k} P_{j i}^{*}\right) \text {. }
$$

From (1) and (2) we have

$$
{ }_{k} P_{i j}^{*}=\frac{{ }_{k} F_{i j}^{*}}{{ }_{j} F_{j k}^{*}} .
$$

Suppose $j \neq k$. Let $v^{\prime}$ be the smallest value of $v>v_{1}$ for which there exist $n_{1}$ and $n_{2}$ such that $v_{1}<n_{1} \leqq v^{\prime}, v_{1}<n_{2} \leqq v^{\prime}$ and $X_{n_{1}}=j, X_{n_{2}}=k$. The expectation $E\left(v^{\prime}-v_{1}\right)$ may be suggestively denoted by $m(i, j$ and $k)$. Recalling that ${ }_{k} F_{i j}^{*}$ is the probability, starting from $i$, of reaching $j$ before $k$, it is not difficult to see that

$$
m(i, j \text { and } k)=m_{i j}+{ }_{k} F_{i j}^{*} m_{j k}=m_{i k}+{ }_{j} F_{i k}^{*} m_{k j} .
$$

Since in a recurrent class ${ }_{k} F_{v}^{*}+{ }_{j} F_{i k}^{*}=1$ we deduce from (4) that

$$
m_{i k}+m_{k j}-m_{i j}={ }_{k} F_{i j}^{*}\left(m_{j k}+m_{k j}\right) \text {. }
$$

Replacing both $i$ and $k$ by $j$ and $j$ by $k$ in (5), we have

$$
m_{j i}={ }_{j} F_{j k}^{*}\left(m_{k j}+m_{j k}\right) \text {. }
$$

From (3), (5), and (6) we obtain then

$$
{ }_{k} P_{i j}^{*}=\frac{m_{i k}+m_{k i}-m_{i j}}{m_{i j}} .
$$

This is the main formula.

Summing (7) over $j$ we obtain

$$
\begin{aligned}
\sum_{j=0}^{\infty} \frac{m_{i k}+m_{k j}-m_{i j}}{m_{i j}} & =\sum_{j=0}^{\infty}{ }_{k} P_{i j}^{*}=\sum_{j=0}^{\infty} \sum_{n=1}^{\infty}{ }_{k} P_{i j}^{(n)} \\
& =\sum_{n=1}^{\infty} \sum_{v=n}^{\infty} F_{i k}^{(v)}=m_{i k .} .
\end{aligned}
$$

Thus (C) is proved. Note that the $m_{j i}$ 's on both sides cancel out.

To prove (A) and (B) we introduce new random variables $X_{n}^{\prime}, n \geqq 1$, as follows

$$
X_{n}^{\prime}=\left\{\begin{array}{cl}
0 & \text { if } X_{v}=i \text { for some } v: 1<v<n ; \\
f\left(X_{n}\right) & \text { if } X_{v} \neq i \text { for all } v: 1 \leqq v<n .
\end{array}\right.
$$

We have, if the series involved converge absolutely: 


$$
\begin{aligned}
E\left\{\sum_{v=v_{1}+1}^{v_{2}} f\left(X_{v}\right)\right\} & =E\left\{\sum_{n=1}^{\infty} X_{n}^{\prime} \mid X_{0}=i\right\}=\sum_{n=1}^{\infty} E\left\{X_{n}^{\prime} \mid X_{0}=i\right\} \\
& =\sum_{n=1}^{\infty} \sum_{j=0}^{\infty}{ }_{i} P_{i j}^{(n)} f(j)=\sum_{j=0}^{\infty}{ }_{i} P_{i j}^{*} f(j)=\sum_{j=0}^{\infty} \frac{m_{i i}}{m_{j j}} f(j)
\end{aligned}
$$

by (7) with $k=i$. Thus (A) is proved.

Similarly, we have

$$
E\left\{\left[\sum_{v=v_{1}+1}^{v_{2}} f\left(X_{v}\right)\right]^{2}\right\}=E\left\{\sum_{n=1}^{\infty}\left[X_{n}^{\prime 2}+2 \sum_{r=1}^{\infty} \sum_{s=r+1}^{\infty} X_{r}^{\prime} X_{s}^{\prime}\right] \mid X_{0}=i\right\} .
$$

As before,

$$
E\left\{\sum_{n=1}^{\infty}{X_{n}^{\prime 2}}^{\prime 2} X_{0}=i\right\}=\sum_{j=0}^{\infty} \frac{m_{i i}}{m_{j j}} f^{2}(j)
$$

Next we have

$$
\begin{aligned}
\sum_{r=1}^{\infty} \sum_{s=r+1}^{\infty} E\left(X_{r}^{\prime} X_{s}^{\prime} \mid X_{0}=i\right) & =\sum_{r=1}^{\infty} \sum_{s=r+1}^{\infty} \sum_{j=0, j \neq i}^{\infty} \sum_{k=0}^{\infty}{ }_{i} P_{i j}^{(r)} f(j){ }_{i} P_{j k}^{(s-r)} f(k) \\
& =\sum_{j=0, j \neq i}^{\infty}{ }_{i} P_{i j}^{*} f(j) \sum_{k=0}^{\infty}{ }_{i} P_{j k}^{*} f(k) .
\end{aligned}
$$

Substituting (9) and (10) into (8) and using (7) we obtain (B).

In a recurrent class we have (Theorem 1 of [1]):

$$
0<{ }_{i} P_{i j}^{*}=\lim _{n \rightarrow \infty} \frac{\sum_{v=0}^{n} P_{i j}^{(v)}}{\sum_{v=0}^{n} P_{i i}^{(v)}}<\infty .
$$

Proof. That $0<{ }_{i} P_{i j}^{*}<\infty$ follows from (3). Now $P_{i j}^{(n)}=\sum_{i=0}^{n} P_{i j}^{(v)}{ }_{i} P_{i j}^{(n-v)}$ where ${ }_{i} P_{i j}^{(0)}=0$. Summing over $n$,

$$
\sum_{n=0}^{N} P_{i j}^{(n)}=\sum_{v=0}^{N} P_{i i}^{(v)} \sum_{n=0}^{N-v}{ }_{i} P_{i j}^{(n)}
$$

Since $\lim _{N \rightarrow \infty} \sum_{n=0}^{N-0}{ }_{i} P_{i j}^{(n)}={ }_{i} P_{i j}^{*}$, it follows easily that

$$
\lim _{N \rightarrow \infty} \frac{\sum_{n=0}^{N} P_{i j}^{(n)}}{\sum_{v=0}^{N} P_{i i}^{(v)}}={ }_{i} P_{i j}^{*} .
$$

On the other hand it is trivial that 


$$
\lim _{N \rightarrow \infty} \frac{\sum_{n=0}^{N} P_{i j}^{(n)}}{\sum_{n=0}^{N} P_{j i}^{(n)}}=1 .
$$

Thus (D) is proved.

\section{REFERENCES}

1. K. L. Chung, Contribution to the theory of Markov chains I, Journal of Research of the National Bureau of Standards vol. 50(1953) pp. 203-208.

2. K. L. Chung and W. H. J. Fuchs, On the distribution of values of sums of random variables, Memoirs of the American Mathematical Society, no. 6, 1951, 12 pp.

3. W. Doblin, Sur deux problèmes de M. Kolmogoroff concernant les chaines dénombrables, Bull. Soc. Math. France vol. 52 (1938) pp. 210-220.

4. —, Thèse, Paris (printed in Bucharest), 1938.

5. J. L. Doob, The law of large numbers for continuous stochastic processes, Duke Math. J. vol. 6 (1940) pp. 290-306.

6. - Stochastic processes, New York, Wiley, 1953.

7. P. Erdös and M. Kac, On certain limit theorems of the theory of probability, Bull. Amer. Math. Soc. vol. 52 (1946) pp. 292-302. 1950.

8. W. Feller, An introduction to probability theory and its applications, New York, Wiley,

9. T. E. Harris, First passage and recurrence distributions, Trans. Amer. Math. Soc. vol. 73 (1952) pp. 471-486.

10. P. Hartman and A. Wintner, On the law of the iterated logarithm, Amer. J. Math. vol. 63 (1941) pp. 169-176.

11. E. Hopf, Ergodentheorie, Ergebnisse der Mathematik, vol. 5, no. 2, 1937.

12. A. N. Kolmogorov, Anfangsgründe der Theorie der Markoffschen Ketten mit unendlichen vielen möglichen Zuständen, Mat. Sbornik N.S. vol. 1 (1936) pp. 607-610; Bull. de l'Univ. d'Etat Moscou (in Russian) vol. 1 (1937). For an exposition in English see Chapter 1 of K. L. Chung, Lecture notes on Markov chains, Columbia Graduate Mathematical Statistics Society, New York, 1951.

13. - A local limit theorem for classical Markov chains (in Russian), Izvestiya Akad. Nauk SSSR. Ser. Mat. vol. 13 (1949) pp. 281-300.

14. P. Lévy, Systèmes markoviens et stationnaires; cas dénombrable, Ann. Ecole Norm. (3) vol. 68 (1952) pp. 327-381.

15. - Théorie de l'addition des variables aléatoires, Paris, Gauthier-Villars, 1937.

16. K. Yosida and S. Kakutani, Markoff process with an enumerable infinite number of states, Jap. J. Math. vol. 16 (1939) pp. 47-55.

17. T. E. Harris and H. E. Robbins, Ergodic theory of Markov chains admitting an infinite invariant measure, Proc. Nat. Acad. Sci. U.S.A. vol. 39 (1953) pp. 860-864.

Cornell University, ITHACA, N. Y.

SyRACUSE UNIVERSITY, SyRaCUSE, N. Y. 